\title{
Modelling Leaky Waves in Planar Dielectric Waveguides
}

\author{
Edik Ayryan $^{1 \star}$, Dmitry Divakov ${ }^{2, \star \star}$, Alexandre Egorov ${ }^{3, \star \star \star}$, \\ Konstantin Lovetskiy ${ }^{2, \star \star \star \star}$, and Leonid Sevastianov $v^{1,2, \dagger}$ \\ ${ }^{1}$ Joint Institute for Nuclear Research, Joliot-Curie 6, 141980 Dubna, Moscow Region, Russia \\ ${ }^{2}$ Peoples' Friendship University of Russia (RUDN University), Miklukho-Maklaya 6, 117198 Moscow, Russia \\ ${ }^{3}$ Prokhorov General Physics Institute, Russian Academy of Sciences, Vavilova 38, 119991 Moscow, Russia
}

\begin{abstract}
Experimentally observed leaky modes of a dielectric waveguide are characterised by a weak tunnelling of the light through the waveguide and its long-time propagation along the waveguide. Traditional mathematical models of leaky waveguide modes meet some contradictions resolved using additional considerations. We propose a model of leaky modes in a waveguide free from the above contradictions, akin to the quantum mechanical model of the "pseudo-stable" Gamow-Siegert states.

By separating variables, from the complete problem for plane inhomogeneous waves we obtain a non-self-adjoint Sturm-Liouville problem to determine the complex coefficient of the phase delay of the studied mode. The solution of the complete wave problem determines the propagation cone for the leaky mode of the waveguide, inside which there are no contradictions. Thus, solution is in qualitative agreement with experimental data.
\end{abstract}

\section{Introduction}

In the second half of the 20th century, a large number of papers (see references in [1]) were published on the theoretical description and methods for computing the leaky modes of fibre and planar dielectric waveguides. In this case, the problem of description and numerical determination of the spectral parameters of the outgoing waves was solved successfully both theoretically and numerically. The solution of the problem of describing and modelling the electromagnetic field of these waves encountered certain difficulties. Formal mathematical solutions of Maxwell equations and/or Helmholtz equations that follow from them for individual modes exhibited exponential growth with the distance increase from the waveguiding core. In other words, the mathematical solutions obtained were nonphysical. Physical considerations for the "correct behaviour" of real leaky modes were proposed, but they could not be included in the computational algorithms for computer simulation. The exceptions were additional barriers modelled by so-called W waveguides (ARROW waveguides).

In this paper, we propose to obtain solutions of boundary value problems for wave equations with asymptotic conditions of Siegert outgoing waves using a numerical method. The numerical solutions

\footnotetext{
${ }^{\star}$ e-mail: ayrjan@jinr.ru

$\star \star$ e-mail: divakov_dv@rudn.ru

$\star \star \star$ e-mail: yegorov@kapella.gpi.ru

$\star \star \star \star$ e-mail: lovetskiy_kp@rudn.ru

†e-mail: sevastianov_la@rudn.ru
} 
obtained in this way are alike to the solutions reported in Ref. [2], but additionally allow one to describe phase fronts of the outgoing waves and "angular outflow cones".

In Ref. [3], it was noted that in most publications on leaky modes there are no field plots of different types of leaky modes calculated by numerical methods. In this case, the authors of a number of publications (e.g., [4]) propose to replace the resulting modes with radiative ones in limited domains. Our studies have shown that this can lead, first, to a large error in the calculation of losses, and second, to inaccurate values of the field profiles of leaky modes at distances exceeding several wavelengths $\left(\geq 2 \lambda_{0}\right)$ of the used electromagnetic radiation [3]. The replacement of one wave by another sometimes used in this case requires serious analysis in each particular case.

\section{Problem setting for mathematical modelling of leaky modes in symmetric dielectric waveguides}

Consider a symmetric three-layer planar waveguide $(-\infty<x<+\infty,-\infty<y<+\infty,-\infty<$ $z<+\infty)$ consisting of a dielectric film $(0<x<h)$ of thickness $h$ with a real refractive index $n_{f}$ surrounded by two cover layers $(x<0$ and $x>h)$ with a real refractive index $n_{c}<n_{f}$. The waveguide is homogeneous in $y O z$ plane and the positive direction of $z$-axis is the direction along which the waveguide modes travel. The generally accepted model of the electromagnetic field in a planar region (infinitely extended along the axis $O y$ ) are fields that are independent of the variable $y$. In this case, the system of Maxwell equations is divided into two independent subsystems for the so-called TE-modes and for the TM-modes.

In contrast to the traditional representation of subsystems using the Helmholtz equations, our main feature is that we reduce Maxwell's equations to subsystems with wave equations in order to consider the leaky modes as a wave process. The subsystem for the TE-mode can be presented as a single wave equation for the master component $E_{y}$ and boundary conditions of "outgoing waves" corresponding to the Gamow-Siegert model [2, 5-7]

$$
\left\{\begin{array}{l}
\frac{\partial^{2} E_{y}}{\partial x^{2}}+\frac{\partial^{2} E_{y}}{\partial z^{2}}-\frac{n^{2}(x)}{c^{2}} \frac{\partial^{2} E_{y}}{\partial t^{2}}=0 \\
{\left[\frac{\partial E_{y}}{\partial x}-i k_{0} p_{c}(\beta) E_{y}\right]_{x=h}=0, \quad\left[\frac{\partial E_{y}}{\partial x}+i k_{0} p_{c}(\beta) E_{y}\right]_{x=0}=0} \\
\left.E_{y}(x, z, t)\right|_{z=0, t=0}=E_{y}^{0}(x),
\end{array}\right.
$$

where $p_{c}(\beta)=\sqrt{n_{c}^{2}-\beta^{2}}, \beta$ is the phase delay coefficient, $k_{0}$ is the wavenumber. The subsystem for the TE mode also includes two equations that relate the components $H_{x}, H_{z}$ to the master component $E_{y}$. The mathematical model described by Eqs. (1) allows the separation of variables in (1) and yields solutions corresponding to the leaky modes propagating in the positive direction of the $z$-axis:

$$
\begin{gathered}
\left.E_{y j}^{+}\right|_{x>h}=A_{c j}^{+} \cdot \exp \left[i k_{0} x \sqrt{n_{c}^{2}-\beta_{j}^{2}}+i k_{0} \beta_{j} z-i \omega t\right], \\
\left.E_{y j}^{+}\right|_{\substack{x<h, x>0}}=A_{f j}^{+} \cdot \exp \left[i k_{0} x \sqrt{n_{f}^{2}-\beta_{j}^{2}}+i k_{0} \beta_{j} z-i \omega t\right]+B_{f j}^{+} \cdot \exp \left[-i k_{0} x \sqrt{n_{f}^{2}-\beta_{j}^{2}}+i k_{0} \beta_{j} z-i \omega t\right], \\
\left.E_{y j}^{+}\right|_{x<0}=B_{c j}^{+} \cdot \exp \left[-i k_{0} x \sqrt{n_{c}^{2}-\beta_{j}^{2}}+i k_{0} \beta_{j} z-i \omega t\right],
\end{gathered}
$$


where $\omega$ is the frequency, and $\beta_{j}$ are the eigenvalues of the non-self-adjoint Sturm-Liouville problem with the boundary conditions that separate the leaky modes $[4,8]$

$$
\left\{\begin{array}{l}
X^{\prime \prime}+k_{0}^{2} n_{f}^{2} X=k_{0}^{2} \beta^{2} X, \\
X^{\prime}(0)+i k_{0} X(0) \sqrt{n_{c}^{2}-\beta^{2}}=0, \quad X^{\prime}(h)-i k_{0} X(h) \sqrt{n_{c}^{2}-\beta^{2}}=0 .
\end{array}\right.
$$

We solve the spectral problem for the non-self-adjoint differential operator (5) by the numeric method described in [9]. The eigenvalues corresponding to the leaky modes are localised in the first quadrant $\operatorname{Re}(\beta)>0, \operatorname{Im}(\beta)>0$ of the complex plane; moreover $0<\operatorname{Re}(\beta)<n_{c}$ (see [4, 10], as well as [9]). We will consider the complex quantities $p_{c j}=\sqrt{n_{c}^{2}-\beta_{j}^{2}}=\operatorname{Re}\left(p_{c j}\right)+i \operatorname{Im}\left(p_{c j}\right), p_{f j}=$ $\sqrt{n_{f}^{2}-\beta_{j}^{2}}=\operatorname{Re}\left(p_{f j}\right)+i \operatorname{Im}\left(p_{f j}\right)$ and $\beta_{j}=\operatorname{Re}\left(\beta_{j}\right)+i \operatorname{Im}\left(\beta_{j}\right)$ in the solutions explicitly using their real and imaginary parts. The angular region around the $z$-axis inside which leaky modes with nonincreasing amplitude exist is pinpointed in Fig. 1 by light color. The first three values of the complex phase delay coefficient $\beta$ and $p_{c}$ are

$$
\begin{array}{ll}
\beta_{1}=1.40442+0.16876 i, & p_{c 1}=0.60758-0.39010 i, \\
\beta_{2}=1.15214+0.49548 i, & p_{c 2}=1.15106-0.49595 i, \\
\beta_{3}=0.91446+1.03205 i, & p_{c 3}=1.64850-0.57250 i,
\end{array}
$$

calculated for a waveguide with $n_{c}=1.47, n_{f}=1.565, \lambda=0.55 \mu \mathrm{m}$ and $h=1.1 \lambda$.

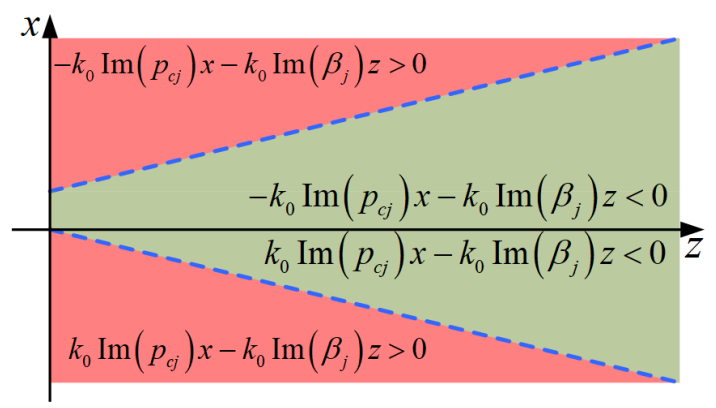

Figure 1. (Colour online) Domains of amplitude increase/attenuation and the line of constant amplitude for an inhomogeneous plane wave in the $x O z$-plane

For clarity, in Fig. 2 we present the instantaneous distribution of the field strength for the leaky mode, corresponding to the first complex eigenvalue. The calculated amplitude profile is in qualitative agreement with the data reportid in Fig. 13 of Ref. [11]. All the calculations and the plots shown in Fig. 2 have been conducting using Maple [12].

\section{Conclusion}

The proposed model provides a sustainable design of integrated optical structures based on a threelayer waveguide.

In this paper, we proposes a "complete" mathematical model of the leaky modes of a planar threelayer dielectric waveguide. We have established the region of existence of leaky modes corresponding 


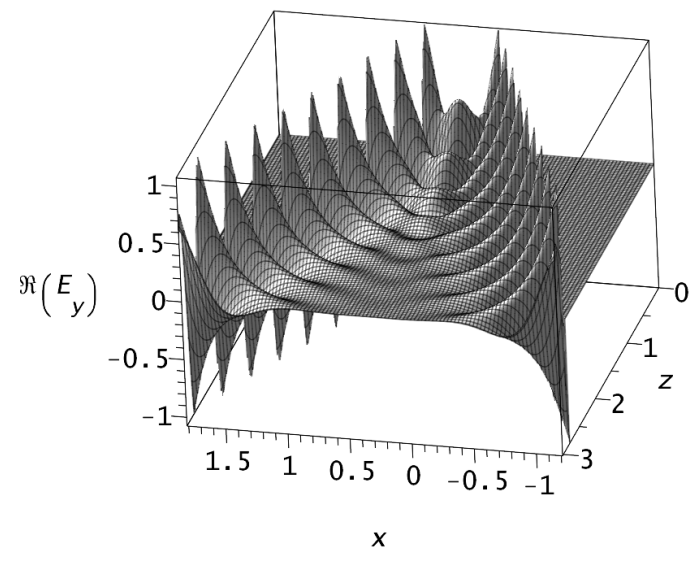

(a)

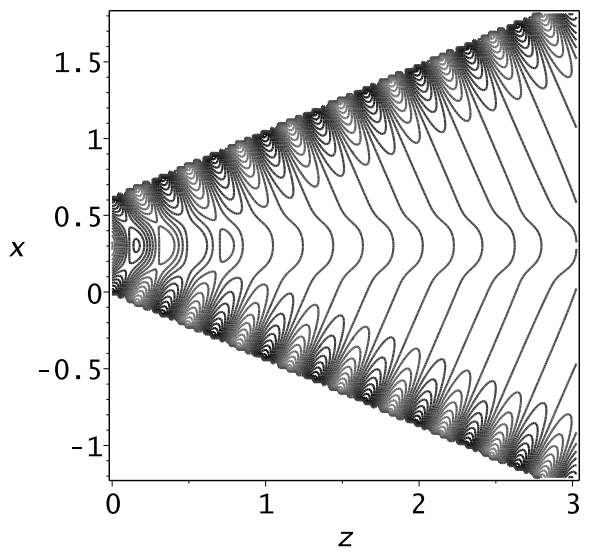

(b)

Figure 2. Instantaneous amplitude distribution in the $x O z$ plane for the leaky mode corresponding to the first complex value presented as a 3D plot (a) and as a contour map (b).

to inhomogeneous waves with non-increasing amplitude. If the wave vector of an inhomogeneous wave is located inside an angular region around the $z$-axis, then such a flowing mode has a limited amplitude and it can propagate over a sufficiently long distance along the waveguide keeping a significantly large amplitude (i.e., without extinction).

\section{Acknowledgement}

The publication funded by RFBR according to the research projects No. 18-07-00567, No. 18-5118005 and No. 19-01-00645.

\section{References}

[1] J. Hu, C.R. Menyuk, Advances in Optics and Photonics 1, 1, 58-106 (2009)

[2] A.J.F. Siegert, Phys. Rev. 56, 750-752 (1939)

[3] A.A. Egorov, Quantum Electronics 42, 4, 337-344 (2012)

[4] E.I. Golant, K.M. Golant, Technical Physics 51, 8, 1060-1068 (2006)

[5] G. Gamow, Z. Phys. 51, 204-212 (1928)

[6] R. de la Madrid, Journal of Mathematical Physics 53, 102113 (2012)

[7] O. Rosas-Ortiz, N. Fernandez-Garcia, S. Cruz y Cruz, AIP Conf. Proc. 1077, 31 (2008)

[8] D. Stowell, J. Tausch, PIERS Online 6, 7, 669-673 (2010)

[9] D. Divakov, A. Tiutiunnik, A. Sevastianov, MATEC Web of Conferences 186, 01009 (2018)

[10] J. Zhu, Ya.Ya. Lu, Journal of Lightwave Technology 24, 3, 1619-1623 (2006)

[11] C. Fernández, J. Monzó-Cabrera, IEEE Trans. on Antennas and Propagation 61, 6, 2981-2990 (2013)

[12] Maple for Education \& Research, https://www.maplesoft.com/MapleEducation/ 